\title{
DIAGNÓSTICOS DE ENFERMAGEM MAIS UTILIZADOS EM SERVIÇO DE EMERGÊNCIA*
}

Meiry Fernanda Pinto Okuno', Natalha da Costaㄹ, Maria Carolina Barbosa Teixeira Lopes ${ }^{3}$, Cássia Regina Vancini Campanharo', Ruth Ester Assayag Batista ${ }^{4}$

${ }^{1}$ Enfermeira. Doutora em Ciências. Escola Paulista de Enfermagem da Universidade Federal de São Paulo. São Paulo-SP-Brasil. ${ }^{2}$ Enfermeira. Especialista em Enfermagem em Emergência. Associação Saúde da Família. São Paulo-SP-Brasil.

${ }^{3}$ Enfermeira. Mestre em Ciências. Escola Paulista de Enfermagem da Universidade Federal de São Paulo. São Paulo-SP-Brasil. ${ }^{4}$ Enfermeira. Doutora em Ciências. Professora da Universidade Federal de São Paulo. São Paulo-SP-Brasil.

RESUMO: Objetivou-se identificar os principais diagnósticos de enfermagem utilizados por experts em área de emergência. Estudo descritivo realizado em Serviço de Emergência, período de junho a novembro de 2011. Utilizouse a Técnica Delphi para a obtenção do consenso entre sete profissionais de diferentes instituições brasileiras, sobre os diagnósticos de enfermagem prevalentes em Serviços de Urgência e Emergência. Os diagnósticos de enfermagem que tiveram consenso foram: Troca de gases prejudicada, Padrão respiratório ineficaz, Ventilação espontânea prejudicada, Risco de infecção, Risco de integridade da pele prejudicada, Integridade tissular prejudicada e Risco de quedas. Conclui-se que a Técnica Delphi mostrou-se como grande facilitadora para a obtenção de consenso, sobre os diagnósticos de enfermagem prevalentes em Serviços de Emergência, relacionados aos domínios da North American Nursing Diagnosis Association de "segurança e proteção", "atividade e repouso" e "eliminação e troca". DESCRITORES: Diagnóstico de enfermagem; Enfermagem em emergência; Cuidados de enfermagem.

\section{THE MOST USED NURSING DIAGNOSES AT AN EMERGENCY SERVICE}

\begin{abstract}
The objective was to identify the main nursing diagnoses used by experts in the emergency area. A descriptive study was undertaken at an emergency service between June and November 2011. The Delphi technique was used to reach a consensus among seven professionals from different Brazilian institutions about the prevailing nursing diagnoses at emergency care services. A consensus was reached on the following nursing diagnoses: Impaired gas exchange, Ineffective breathing pattern, Impaired spontaneous ventilation, Risk for infection, Risk for impaired skin integrity, Impaired tissue integrity and Risk for falls. In conclusion, the Delphi technique showed to be a great facilitator to reach a consensus about the prevailing nursing diagnoses at emergency care services related to the "safety and protection", "activity and rest" and "elimination and exchange" domains of the North American Nursing Diagnosis Association.
\end{abstract}

DESCRIPTORS: Nursing diagnosis; Emergency nursing; Nursing care.

\section{DIAGNÓSTICOS DE ENFERMERÍA MÁS UTILIZADOS EN SERVICIO DE EMERGENCIA}

RESUMEN: Fue objetivo del estudio identificar los principales diagnósticos de enfermería utilizados por experts en área de emergencia. Estudio descriptivo realizado en Servicio de Emergencia, periodo de junio a noviembre de 2011. Se utilizó la Técnica Delphi para obtener consenso entre siete profesionales de distintas instituciones brasileñas acerca de los diagnósticos de enfermería prevalentes en Servicios de Urgencia y Emergencia. Los diagnósticos de enfermería que tuvieron consenso fueron: Cambio de gases perjudicado, Patrón respiratorio ineficaz, Ventilación espontánea perjudicada, Riesgo de infección, Riesgo de integridad de la piel perjudicada, Integridad tisular perjudicada y Riesgo de caídas. Se concluye que la Técnica Delphi se mostró como gran facilitadora para obtener consenso acerca de los diagnósticos de enfermería prevalentes en Servicios de Emergencia, relacionados a los dominios de la North American Nursing Diagnosis Association de "seguridad y protección", "actividad y reposo" u "eliminación y cambio". DESCRIPTORES: Diagnóstico de enfermería; Enfermería en emergencia; Cuidados de enfermería.

\footnotetext{
*Artigo extraído da Monografia do Programa de Residência Multiprofissional em Urgência e Emergência, intitulada: "O uso da Técnica Delphi para identificação dos diagnósticos de enfermagem prevalentes no Serviço de emergência". Universidade Federal de São Paulo, 2011.
}

Autor Correspondente:

Cássia Regina Vancini Campanharo

Universidade Federal de São Paulo

Rua Napoleão de Barros, 754 - 04024-002 - São Paulo-SP-Brasil

E-mail: vcassia@hotmail.com
Recebido: $28 / 11 / 2014$

Finalizado: 26/04/2015 


\section{INTRODUÇÃO}

A procura pelos serviços de emergência aumentou exponencialmente nos últimos 30 anos, associada à maior complexidade e gravidade dos pacientes. Neste contexto, a superlotação destes serviços tem sido um assunto muito discutido na literatura como forma de caracterizar a causa deste fenômeno e encontrar meios para solucionar ou amenizar este problema ${ }^{(1)}$.

Adicionalmente, fatores estressantes enfrentados pela equipe de enfermagem nos serviços de emergência como o número de funcionários insuficiente; falta de respaldo institucional e profissional; carga de trabalho elevada; necessidade da realização de procedimentos médico e de enfermagem em curto período de tempo; espaço físico inadequado; relacionamentos conflituosos no ambiente de trabalho com profissionais, familiares e usuários; dentre outros contribuem para assistência de enfermagem muitas vezes pouco segura, capaz de lesar os usuários, que têm direito a atendimento livre de riscos, causando grande impacto sobre o cuidado prestado ${ }^{(2)}$.

Neste cenário, não apenas se discute o perfil do enfermeiro emergencista, mas também o funcionamento do sistema de saúde, que permite a superlotação destes setores e não fornece recursos humanos e materiais suficientes para proporcionar ao paciente assistência segura e de qualidade $^{(3)}$.

Durante o atendimento às emergências clínicas e cirúrgicas, as intervenções médicas são prioridade para o restabelecimento das funções vitais do paciente, porém, depois de estabelecidas, mesmo com persistência da gravidade, faz-se necessário o suprimento de outras necessidades do indivíduo. Observase, ainda hoje, que o modelo biomédico é predominante na assistência à saúde, no qual o paciente é visto como um conjunto de órgãos que podem adoecer e, quando isto acontece, prevalece uma intervenção isolada, com objetivo de cura imediata, desvalorizando a globalidade da pessoa $^{(4)}$.

O cuidado de enfermagem ainda é influenciado pelo modelo assistencial hospitalocêntrico, e fortemente centrado na doença e não no ser humano ${ }^{(5)}$. Nesse contexto o processo de enfermagem se faz importante, pois é a concretização de um método de trabalho elaborado para nortear o cuidado do indivíduo na sua integralidade(6). Quanto mais crítica é a situação do paciente, mais importante e necessário é sistematizar a assistência de enfermagem. A utilização de um método de trabalho é indispensável para que as ações sejam organizadas e o atendimento atenda as necessidades dos pacientes ${ }^{(7)}$.

Na Resolução do COFEN no 358 de 15 de outubro de 2009, o Conselho Federal de Enfermagem institui a necessidade de implementação do Processo de Enfermagem, nos serviços de saúde, sendo está uma atividade privativa dos enfermeiros, para que se obtenha um cuidado de enfermagem seguro e humanizado às exigências do paciente ${ }^{(8)}$.

O modelo de diagnóstico de enfermagem recomendado pela North American Nursing Diagnosis Association (NANDA) ${ }^{(9)}$, associação norte americana que congrega estudos e pesquisas sobre diagnósticos de enfermagem, fornece linguagem padronizada, utilizada como produto do raciocínio e julgamento clínico sobre as respostas humanas aos problemas de saúde e processos vitais essenciais ao paciente crítico $^{(10)}$.

A superação do modelo biomédico é fundamental para assistência de enfermagem prestada ao paciente no Serviço de Emergência, por isso objetivou-se neste estudo identificar os principais diagnósticos de enfermagem utilizados por experts na área de emergência, utilizando a Técnica Delphi, com o intuito de direcionar ações que viabilizem a recuperação de conceitos e práticas humanizadoras na assistência de enfermagem ao paciente em situação de emergência.

\section{MÉTODO}

Tratou-se de um estudo do tipo descritivo realizado em um hospital universitário do estado de São Paulo. Foi realizado no período de junho a novembro de 2011, com profissionais de diferentes instituições brasileiras, especialistas no atendimento a pacientes adultos em situação de urgência e emergência.

Utilizou-se a Técnica Delphi, que permite obter consenso de um grupo de experts em determinada área do conhecimento, a respeito de um determinado fenômeno. Os experts são definidos como profissionais efetivamente 
engajados e experientes em suas áreas de atuação ${ }^{(11)}$. Quanto ao número de participantes, a literatura não menciona um número ideal, mas diz que um número mínimo de cinco é suficiente para o controle de concordância, que está entre $50 \%$ e $80 \%{ }^{(11-12)}$.

Para a formação do painel de experts foram contatados 25 especialistas em urgência e emergência do Brasil. Inicialmente buscou-se na Plataforma Lattes por enfermeiros especialistas com os descritores enfermagem e emergência; outra estratégia utilizada foi o convite aos profissionais de dois hospitais públicos e dois hospitais privados da cidade de São Paulo, que atuam na área de urgência.

Os critérios de inclusão para este estudo foram: ser enfermeiro(a); possuir cinco ou mais anos de experiência na assistência direta ao paciente em unidades de urgência e emergência e, ou atuação na docência ou na pesquisa nesta área de concentração; ter experiência com a utilização de diagnósticos de enfermagem e aceitar participar deste estudo.

$\mathrm{O}$ estudo foi dividido em quatro fases. A primeira fase consistiu do envio de carta convite e do termo de consentimento livre e esclarecido, por correio eletrônico. Após recebimento de aceite foram enviados dois formulários para preenchimento. O primeiro com dados demográficos e outro com todos os diagnósticos de enfermagem contemplados na Taxonomia NANDA 2009-2011 ${ }^{(9)}$ com uma escala tipo likert com pontuação de um a quatro, para classificação da prevalência de cada diagnóstico em Serviço de Urgência e Emergência: (1) "Eu uso este diagnóstico em menos de $25 \%$ das situações", (2) "Eu uso este diagnóstico de 25\% a 49\% das situações", (3) "Eu uso este diagnóstico de 50\% a $74 \%$ das situações" e (4) "Eu uso este diagnóstico em $75 \%$ ou mais das situações".

Os participantes incluídos no estudo foram aqueles que devolveram os questionários preenchidos por completo e no prazo estipulado de cinco dias, totalizando sete experts.

A segunda fase foi o armazenamento dos dados obtidos no programa EXCEL® e sua análise quanto à frequência absoluta. Os diagnósticos com médias inferiores a 50\% foram excluídos, valor este estipulado pelos pesquisadores, previamente à coleta de dados.
Na terceira fase, após a exclusão dos diagnósticos de enfermagem com frequência inferior a $50 \%$, as respostas foram reenviadas, por meio de correio eletrônico, ao grupo para reavaliação. Cada experts teve contato com as respostas dos outros juízes, buscando-se alcançar o maior nível de concordância entre os mesmos. $\mathrm{O}$ anonimato dos respondentes foi garantido. Um novo prazo de cinco dias foi estipulado para a devolução dos questionários respondidos.

$\mathrm{Na}$ quarta fase os dados obtidos foram, novamente, tabulados no programa EXCEL $\mathbb{R}$ e analisados quanto à frequência absoluta. Os diagnósticos com média inferior a 50\% foram, mais uma vez, excluídos, sendo que foram considerados, nesta fase, aqueles que obtiveram concordância superior a $70 \%$, percentual este que foi estipulado pelos pesquisadores.

Esteestudo foi desenvolvidoapósanáliseeaprovação do Comitê de Ética em Pesquisa da Universidade Federal de São Paulo (Protocolo nº 0640/11).

\section{RESULTADOS}

Os sete profissionais incluídos no estudo eram todos do sexo feminino, com média de idade de 33,7 anos e pertencentes a três estados: São Paulo (71,4\%), Paraná (14,3\%) e Rio Grande do Sul (14,3\%).

$\mathrm{A}$ área de atuação dos experts foi assistencial (28,4\%), assistencial/pesquisa (28,4\%), assistencial/ ensino/pesquisa (14,4\%), ensino/pesquisa (14,4\%), ensino/pesquisa/gestão (14,4\%). As instituições nas quais eles desenvolviam suas atividades foram: instituição pública $(71,4 \%)$, instituição privada $(14,3 \%)$ e uma instituição que recebia subsídios públicos e privados (14,3\%). Quanto ao porte das instituições, a maioria deles $(71,4 \%)$ desempenhava suas atividades em hospitais de grande porte e apenas dois em instituições de médio porte $(28,6 \%)$. Quanto ao tipo de demanda destas instituições, $100 \%$ dos experts responderam que as instituições as quais pertenciam eram de demanda espontânea e referenciada.

O tempo de experiência dos participantes na área de urgência e emergência foi de cinco a nove anos (71,2\%) e nove anos ou mais (28,8\%). Os diagnósticos de enfermagem mais prevalentes nos Serviços de Urgência e Emergência relatados pelos experts estão apresentados na Tabela 1. 
Tabela 1 - Diagnósticos de enfermagem mais prevalentes em Serviço de Urgência e Emergência, segundo experts na área. São Paulo, SP, Brasil, 2011

\begin{tabular}{lcc}
\hline Diagnósticos & $\mathbf{n}$ & $\mathbf{\%}$ \\
\hline Troca de Gases Prejudicada & 5 & 71,4 \\
\hline Padrão Respiratório Ineficaz & 6 & 85,7 \\
\hline Ventilação Espontânea Prejudicada & 5 & 71,4 \\
\hline Risco de Infecção & 6 & 85,7 \\
\hline $\begin{array}{l}\text { Risco de Integridade da Pele } \\
\text { Prejudicada }\end{array}$ & 6 & 85,7 \\
\hline Integridade Tissular Prejudicada & 5 & 71,4 \\
\hline Risco de Quedas & 5 & 71,4 \\
\hline
\end{tabular}

Os diagnósticos de enfermagem que tiveram consenso entre os experts superior a $70 \%$ foram $57,1 \%$ do domínio da NANDA de "segurança e proteção", seguido por $28,6 \%$ do domínio "atividade e repouso" e 14,3\% do domínio "eliminação e troca".

\section{DISCUSSÃO}

O objetivo deste estudo foi identificar os principais diagnósticos de enfermagem utilizados nos Serviços de Emergência, por meio da opinião de especialistas, com experiência na área de urgência e emergência, utilizando a Técnica Delphi. Acredita-se que a identificação pode contribuir na atuação dos enfermeiros destas unidades que atualmente lidam com o desafio de gerenciar a superlotação destes serviços e manter a qualidade da assistência ${ }^{(13)}$.

Neste estudo os diagnósticos Troca de Gases Prejudicada, Ventilação Espontânea Prejudicada e Padrão Respiratório Ineficaz foram os mais frequentes. Comparando-se com o achado de estudo desenvolvido em Minas Gerais, que associou o Protocolo de Manchester com os Diagnósticos de Enfermagem, também se encontrou no nível I de prioridade os diagnósticos de enfermagem associados à função respiratória como prevalente, padrão respiratório ineficaz e troca de gases prejudicada, porém a dor aguda $(65,0 \%)$ foi o diagnóstico mais frequente ${ }^{(14)}$.

Neste estudo os três diagnósticos de enfermagem direcionados para a função respiratória dos indivíduos, provavelmente estão associados à característica do atendimento de emergência, que é tratar as causas que ameaçam a vida. Nestas unidades é alta a prevalência da alteração da função respiratória, que pode estar associada às manifestações clínicas de alterações da oxigenação por eventos pulmonares ou não, que causam problema real ou potencial à saúde e fazem o cliente buscar atendimento ${ }^{(15)}$.

Os pacientes com distúrbios respiratórios exigem que o enfermeiro tenha conhecimentos específicos para que, por meio do raciocínio clínico, identifique precocemente situações de risco e proponha intervenções imediatas para preservar e salvar vidas. O enfermeiro é o profissional, que permanece maior tempo ao lado do paciente, sendo responsável pela monitorização e observação constante destes indivíduos ${ }^{(16)}$.

Nesta pesquisa, os diagnósticos de enfermagem Risco de infecção, Risco de integridade da pele prejudicada, Integridade tissular prejudicada e Risco de quedas, também, tiveram consenso entre os experts. A prevalência do diagnóstico Risco de infecção pode estar relacionada aos procedimentos invasivos e imunossupressão, muitas vezes, presente no paciente atendido no Serviço de Emergência ${ }^{(16)}$. Os diagnósticos Risco de integridade da pele prejudicada e Integridade tissular prejudicada são prevalentes em pacientes críticos, pois, muitas vezes, necessitam de repouso pela condição clínica em que se encontram e dependem da equipe de enfermagem para mobilização no leito ${ }^{(7)}$. O consenso sobre a prevalência do diagnóstico Risco de quedas pode estar associado ao fato de que muitos pacientes no Serviço de Emergência estão agitados, confusos e agressivos, além de utilizarem medicamentos como benzodiazepínicos e sedativos ou hipnóticos ${ }^{(17)}$.

Os nossos achados corroboram com os resultados de outro estudo realizado em um Serviço de Atenção Móvel de Urgência (SAMU), da Secretaria Municipal de Ribeirão Preto, estado de São Paulo, com pacientes vítimas de trauma, que identificou os seguintes diagnósticos de enfermagem nas subcategorias oxigenação/ respiração, integridade tecidual e integridade física, respectivamente: Desobstrução ineficaz de vias aéreas, Padrão respiratório ineficaz, Risco para aspiração e Risco para função respiratória alterada; Integridade da pele prejudicada e Integridade tissular prejudicada; e Risco de quedas e Risco de infecção ${ }^{(18)}$.

Houve também semelhanças em estudo realizado em um hospital terciário de São Paulo, 
com o objetivo de identificar os diagnósticos de enfermagem associados à ocorrência de óbitos em pacientes de trauma, com problemas de enfermagem relacionados às vias aéreas, ao padrão respiratório e a perfusão de órgãos e sistemas responsáveis por fatores de risco para o óbito dos pacientes. Entretanto, o mesmo estudo evidenciou que os diagnósticos: desobstrução ineficaz de vias aéreas, conforto prejudicado e dor aguda ${ }^{(19)}$ foram fatores de proteção para a ocorrência dos óbitos. Esses achados reafirmam a necessidade de priorizar problemas/ diagnósticos de enfermagem nas situações de emergência, afim de que haja o planejamento das ações e a tomada de decisão imediata frente à complexidade desses pacientes ${ }^{(20)}$.

Nesta pesquisa os diagnósticos de enfermagem que tiveram consenso entre os experts, superior a $70 \%$, foram em sua maioria voltados aos aspectos biomédicos: $(57,1 \%)$ do domínio da NANDA de "segurança e proteção", seguido por 28,6\% do domínio "atividade e repouso" e 14,3\% do domínio "eliminação e troca". Estes resultados corroboram, em parte, com outros dois estudos brasileiros, um realizado em Belo Horizonte em uma Unidade de Emergência ${ }^{(7)}$ e outro com enfermeiros de diversas unidades hospitalares, incluindo Unidades de Terapia Intensiva e o Serviço de Emergência, em âmbito nacional(21), em que os domínios mais representados foram "atividade e repouso", "segurança e proteção", "nutrição" e "enfrentamento". Estes resultados podem estar relacionados à assistência prestada no serviço de emergência, setor que demanda por atendimento aos pacientes críticos, em situação de risco iminente de vida. Exige-se da equipe de enfermagem cuidados complexos, que priorizem a estabilização do paciente e o suporte à vida ${ }^{(22)}$.

Deste modo, o enfermeiro como gerenciador da equipe de enfermagem, deve programar e priorizar o cuidado a ser prestado, considerando as peculiaridades que apresentam os pacientes no Serviço de Emergência e o diagnóstico de enfermagem fornece critérios para a condução da assistência prestada em um panorama em que a tempo entre a vida e a morte é sutil(22).

Além disso, a complexidade dos pacientes atendidos nestes locais, muitas vezes, justifica a permanência de um acompanhante/familiar, para auxiliar nas necessidades do paciente. Esse cenário implica no atendimento não só das necessidades dos pacientes, mas, também, de seus acompanhantes, frequentemente, tomados por medo e ansiedade, decorrentes da situação de saúde do paciente e da ruptura da estrutura familiar. Independente do local de atendimento, a família deve ser entendida como uma extensão do paciente, o que exige adaptação nas ações da equipe de enfermagem para o enfrentamento deste momento ${ }^{(23)}$.

Por fim, a Sistematização da Assistência de Enfermagem vem sendo largamente utilizada nos últimos anos como método científico para instrumentalizar a resolução de problemas dos pacientes e tornar o cuidado individualizado, além de embasar e fundamentar cientificamente as ações do enfermeiro, este processo é considerado como atividade privativa deste profissional de saúde $^{(7)}$. Portanto a criação de protocolos no Serviço de Urgência e Emergência com base nos diagnósticos de enfermagem pode direcionar o julgamento clínico do enfermeiro sobre os problemas de saúde enfrentados pelo paciente crítico, permitindo a agilidade para a elaboração de um plano de cuidado integral ${ }^{(16)}$.

A principal limitação do presente estudo foi a participação de somente experts de três estados brasileiros, ou seja, o consenso sobre os diagnósticos de enfermagem nesta pesquisa talvez fosse diferente se experts de outros estados tivessem participado. De qualquer modo, os resultados deste estudo podem contribuir para a criação de protocolos que estejam ancorados na sistematização da assistência de enfermagem, favorecendo a qualidade e a segurança do cuidado prestado ao paciente no Serviço de Urgência e Emergência.

\section{CONCLUSÃO}

O uso da Técnica Delphi mostrou-se como grande facilitadora para obtenção de consenso sobre determinado assunto. É importante salientar alguns aspectos que dificultaram o estudo como a dificuldade em encontrar os experts para compor o painel, problemas relativos ao retorno de questionários, com a abstenção de percentual de participantes, ou seja, da totalidade dos questionários enviados aos participantes do painel Delphi, alguns não foram devolvidos preenchidos, havendo perda de participantes. 
Sugere-se que mais estudos sejam realizados para identificar os Diagnósticos de Enfermagem mais prevalentes nos Serviços de Urgência e Emergência, a fim de nortear os diagnósticos de enfermagem que facilitem a assistência de enfermagem integral, fornecendo subsídios para a elaboração de planos de cuidados individualizados para pacientes críticos.

\section{REFERÊNCIAS}

1. Anneveld M, Van der Linden C, Grootendorst D, GalliLeslie M. Measuring emergency department crowding in an inner city hospital in The Netherlands. Int J Emerg Med. 20138;6(1):1-7.

2. Gentile S, Vignally P, Durand AC, Gainotti S, Sambuc $\mathrm{R}$, Gerbeaux P. Nonurgent patients in the emergency department? A French formula to prevent misuse. BMC Health Serv Res. 2010;10(66):1-6.

3. Durand AC, Gentile S, Gerbeaux P, Alazia M, Kiegel $P$, Luigi $S$, et al. Be careful with triage in emergency departments: interobserver agreement on 1,578 patients in France. BMC Emerg Med. 2011;11(19):1-10.

4. Souza CC, Toledo AD, Tadeu LFR, Chianca TCM. Classificação de risco em pronto-socorro: concordância entre um protocolo institucional brasileiro e Manchester. Rev. Latino-Am. Enfermagem. [Internet] 2011;19(1):1-8 [acesso em 30 mar 2015]; Disponível: http://www.revistas.usp.br/rlae/article/view/4285/5447

5. Montezelli JH, Peres AM, Bernardino E. Demandas institucionais e demandas do cuidado no gerenciamento de enfermeiros em um pronto socorro. Rev. bras. enferm. 2011;64(2):348-54.

6. Passos SSS, Sadigusky D. Cuidado de Enfermagem ao paciente dependente e hospitalizado. Rev. enferm. UERJ. 2011;19(4):598-603.

7. Salgado PO, Gonçales PC, Dantas RB, Castro MA, Chianca TCM. Diagnósticos de enfermagem em pacientes numa unidade de emergência. Rev enferm UFPE on line - [acesso em 22 de abr 2015]. Disponível: http://www.revista.ufpe.br/revistaenfermagem/index. php/revista/article/view/5851/pdf_5903. 2013;7(1):83-9.

8. Conselho Federal de Enfermagem. Resolução n. ${ }^{\circ}$ 358, de 15 de outubro de 2009. Dispõe sobre a sistematização da assistência de enfermagem e a implementação do processo de enfermagem em ambientes, públicos ou privados, em que ocorre o cuidado profissional de enfermagem. Brasília: COFEN; 2009.

9. North American Nursing Association. Diagnósticos de enfermagem da NANDA: definições e classificação (2009-2011). Porto Alegre: Artmed; 2010.
10. Santos SSC, Silva ME, Pinho LB, Gautério DP, Pelzer MT, Silveira RS. Risco de quedas em idosos: revisão integrativa pelo diagnóstico da North American Nursing Diagnosis Association. Rev. esc. enferm. USP. 2012;46(5):1227-36.

11. Scarparo AF, Laus AM, Azevedo ALCS, Freitas MRI, Gabriel CS, Chaves LDP. Reflexões sobre o uso da Técnica Delphi em pesquisas na Enfermagem. Rev Rene. 2012;13(1):242-51.

12. Lynn MR. Determination and quantification of content validity. Nurs Res. 1986;35(6):382-5.

13. Santos JLG, Lima MADS, Pestana AL, Garlet ER, Erdmann AL. Desafios para a gerência do cuidado em emergência na perspectiva de enfermeiros. Acta Paul. Enferm. 2013;26(2):136-43.

14. Souza CC, Mata LRF, Carvalho EC, Chianca TCM. Diagnósticos de enfermagem em pacientes classificados nos níveis I e II de prioridade do Protocolo Manchester. Rev. esc. enferm. USP. 2013;47(6):1318-24.

15. Gomes ET, Queiroga AV, Papaléo MMM, Bezerra SMMS. Nursing diagnosis applied to a reference service in cardiac emergencies. Rev. enferm. UFPI. 2014;3(2):16-24.

16. Dutra CSK, Silveira LM, Santos AO, Pereira R, Stabile AM. Diagnósticos de enfermagem prevalentes no paciente internado com sepse no Centro de Terapia Intensiva. Cogitare Enferm. 2014;19(4):747-54.

17. Correa AD, Marques IAB, Martinez MC, Laurino PS, Leão ER, Chimentão DMN. Implantação de um protocolo para gerenciamento de quedas em hospital: resultados de quatro anos de seguimento. Rev. esc. enferm. USP. 2012;46(1):67-74.

18. Cyrillo RMZ, Dalri MCB, Canini SRMS, Carvalho EC, Lourencini RR. Diagnósticos de enfermagem em vítimas de trauma atendidas em um serviço préhospitalar avançado móvel. Rev. Eletr. Enf. [Internet] 2009;11(4) ) [acesso em 22 de abr 2015]. Disponível: http://www.fen.ufg.br/revista/v11/n4/pdf/v11n4a06.pdf

19. Sallum AMC, Santos JLF, Lima FD. Diagnósticos de enfermagem em vítimas fatais decorrentes de trauma no cenário da emergência. Rev. Latino-Am. Enfermagem. [Internet] 2012;20(1) [acesso em 22 de abr 2015]. Disponível: http://www.scielo.br/pdf/rlae/ v20n1/pt_02.pdf

20. Sallum AMC, Sousa RMC. Diagnósticos de enfermagem em vítimas de trauma nas primeiras seis horas após o evento. Acta paul. enferm. [Internet]. 2012;25(2) [acesso em 22 de abr 2015]. Disponível: http://dx.doi. org/10.1590/S0103-21002012000200016

21. Chianca TCM, Souza CC, Ercole FF, Rocha AM. Reconhecimento dos diagnósticos de enfermagem da 
Nanda: uma contribuição. Reme. 2007;2(3):233-7.

22. Lins TH, Lima AXBC, Veríssimo RCSS, Oliveira JM. Diagnósticos e intervenções de enfermagem em vítimas de trauma durante atendimento pré-hospitalar utilizando a CIPE®. Rev. Eletr. Enf. 2013;15(1):34-43.

23. Barros LM, Araújo TM, Neri MFS, Soares E, Caetano JA. Internação em uma unidade de emergência hospitalar: vivência. Cogitare Enferm. 2013;18(2):336-43. 